\title{
EFICIÊNCIA PRODUTIVA E REPRODUTIVA EM VACAS LEITEIRAS
}

\author{
PRODUCTIVE AND REPRODUCTIVE EFFICIENCY IN DAIRY COWS
}

\author{
Tisa Echevarria Leite ${ }^{1}$ José Carlos Ferrugem Moraes $^{2}$ Cláudio Alves Pimentel $^{3}$
}

RESUMO

O presente trabalho teve por objetivo avaliar efeitos de transtornos puerperais sobre a eficiência reprodutiva e produtiva de vacas da raça Holandês de uma estação experimental, durante 24 anos. Foram coletados dados produtivos e reprodutivos de 350 vacas. Todos os dados foram submetidos à análise descritiva. As variáveis dependentes: intervalo entre partos $(I E P)$, intervalo parto concepção (IPC), intervalo parto primeiro cio (IPPC), número de crias $(N C)$ e produção de leite $(P L)$ foram submetidas a análise de variância para determinação dos efeitos da ocorrência de transtornos puerperais (aborto, natimorto, distocia e retenção de placenta) e de mastite. A idade ao primeiro cio (IdPC) foi de 29,4 meses, a idade ao primeiro parto (IdPP) de 37,1 meses e a longevidade $(L)$ de 69,7 meses. O IEP apresentou média de 14,6 meses, o IPPC de 97,0 dias e o IPC de 150,7 dias. $O$ intervalo entre cios (IEC) apresentou média de 48,2 dias, sugerindo falhas na detecção de cios. Foi observado efeito significativo $(P<0,05)$ da ocorrência de aborto sobre o IEP, com esse intervalo sendo 258 dias maior em vacas que apresentaram esse transtorno. A ocorrência de distocia $(P<0,05)$ e natimorto $(P<0,01)$ afetou significativamente o IPC, representando aumentos desse intervalo em 82,4 e 136,1 dias, respectivamente. $O$ IPCC foi o índice menos sensível aos efeitos das desordens reprodutivas, não tendo sofrido efeito de nenhum dos transtornos. A PL foi associada à distocia $(P<0,05)$. Os animais que apresentaram distocia produziram $380,5 \mathrm{~kg}$ de leite a menos do que vacas que não a apresentaram. A partir da análise dos resultados, concluiu-se que procedimentos preventivos para controlar a saúde do rebanho, exames clínicos criteriosos de problemas pósparto e o monitoramento de dados com informações precisas para a análise periódica dos índices produtivos e reprodutivos podem contribuir para uma produção leiteira mais racional $e$ econômica.

Palavras-chave: eficiência reprodutiva, pós-parto, gado leiteiro, produção de leite.

\section{SUMMARY}

The present study was conducted to evaluate the effects of post partum disorders on productive and reproductive performance of Holstein cows, from a dairy experimental station, during 24 years. Productive and reproductive data were collected from 350 cows. Analyses of variance was conducted to evaluate the effects of occurrence of post partum disorders (abortion, stillbirth, dystocia, retained placenta) and mastitis on calving interval (IEP), calving to conception interval (IPC), calving to first estrus interval (IPPC), number of estrus before conception $(N C)$ and milk production (PL). The mean age at first estrus was 29.4 months and the mean age at first calving was 37.1 months. Mean IEP was 14.6 months, mean IPPC was 97.04 days and median IPC was 150.71 days. Median interestrus interval was 24 days, suggesting problems in a estrus detection. A significant effect of occurrence of abortion $(P<0.05)$ was observed on IEP. Calving intervals were 258 days longer in the cows that aborted than in the ones that did not abort. The occurrence of stillbirth $(P<0.01)$ and dystocia $(P<0.05)$ significantly increased IEP in 82.4 days and 136.1 days, respectively. The IPPC was not affected by any of the diseases considered. Milk production was affected by dystocia $(P<0.05)$. Cows that had dystocia produced $380.5 \mathrm{~kg}$ less milk than normal calving cows. We concluded that preventive procedures to control herd health, criterious clinical diagnosis of post partum disorders and development of data bases with precise recordings for regular analysis of the productive and reproductive parameters may lead to a more economic dairy production.

Key words: Reproductive efficiency, post partum, dairy cattle, milk production.

\section{INTRODUÇÃO}

$O$ incremento na produção nacional de leite nos últimos 30 anos, de 5 bilhões de litros/ano em 1960, para cerca de 14 bilhões de litros de leite se deveu, principalmente, pelo aumento no número de vacas ordenhadas $(81 \%)$ e não pelo aumento na produtividade (19\%; FERREIRA, 1991). Um baixo desempenho reprodutivo determina menor produção

\footnotetext{
${ }^{1}$ Departamento de Patologia Animal, Faculdade de Veterinária, Universidade Federal de Pelotas (UFPel), 96010-900, CP 354, Pelotas, RS. E-mail: tisa@ufpel.tche.br.. Autor para correspondência.

${ }^{2}$ Pesquisador CPPSUL-EMBRAPA - Bagé, RS.

${ }^{3}$ Professor Titular Departamento de Patologia Animal, Faculdade de Veterinária, UFPel. 
de leite e de terneiros, incremento nas despesas de manutenção com vacas secas, maiores taxas de descarte e maior número de doses de sêmen por concepção. A eficiência reprodutiva de um rebanho é um dos componentes mais importantes na performance econômica de uma propriedade de produção de leite. Segundo GAINES (1994), o ganho potencial resultante do incremento na taxa reprodutiva é cinco vezes maior que o esperado pelo aumento da qualidade do leite e três vezes maior que o esperado pelo melhoramento genético, sendo apenas inferior aos ganhos que podem ser obtidos pela melhoria na nutrição.

A intensificação dos sistemas de produção de leite indica que as vacas devem ser cobertas o mais cedo possível após o parto. No entanto, os melhores índices de fertilidade são obtidos com serviços a partir dos 60 dias pós-parto. Algumas enfermidades puerperais e metabólicas (STEVENSON \& CALL, 1988), bem como deficiente detecção de cio (ESSLEMONT, 1993) podem ser responsáveis por subfertilidade, prolongando o intervalo parto-concepção.

Considerando essa situação, o objetivo deste estudo foi quantificar transtornos reprodutivos em um rebanho de vacas leiteiras no Rio Grande do Sul, e avaliar seus efeitos sobre determinados índices reprodutivos visando proporcionar novas alternativas para a otimização de índices reprodutivos e a produção leiteira.

\section{MATERIAIS E MÉTODOS}

Foram analisados dados produtivos e reprodutivos, coletados de fichas individuais registradas durante 24 anos, de 350 vacas da raça Holandês, criadas no Centro de Pesquisa de Pecuária dos Campos Sulbrasileiros da Empresa Brasileira de Pesquisa Agropecuária (CPPSUL) situado em Bagé, Rio Grande do Sul. O peso mínimo estabelecido para o primeiro serviço (inseminação artificial) das novilhas era de $340 \mathrm{~kg}$. A interrupção da lactação das vacas era realizada 60 dias antes do parto, ou em função de baixa produção de leite. Os animais eram ordenhados através de ordenha mecânica duas vezes ao dia.

As variáveis dependentes consideradas foram divididas em: características reprodutivas, como intervalo parto concepção (IPC), intervalo parto primei- ro cio (IPPC), intervalo entre cios (IEC), intervalo entre partos (IEP), idade ao primeiro parto (IdPP) e idade ao primeiro cio (IdPC); e características produtivas, como produção de leite (PL), calculada com base na produção diária e corrigida para 300 dias, número de crias (NC) e longevidade em anos (L), considerada como a idade em que os animais apresentaram o último parto. Como variáveis independentes foram considerados aborto, natimorto, distocia, retenção de placenta e mastite. Estatísticas descritivas foram geradas para essas variáveis independentes, sendo as variáveis dependentes IEP, IPC e IPPC submetidas também à análise de variância para determinação dos efeitos das variáveis independentes.

Os intervalos entre cios foram divididos em categorias, de acordo com os critérios de GAINES (1994), para que fosse possível a análise do perfil dessa variável. $O$ perfil do intervalo entre cios geralmente é recomendado para analisar a eficiência da detecção de cio e a mortalidade embrionária. As categorias consideradas foram 0-3 dias, 4-17 dias, 18-24 dias, 25-35 dias, 36-48 dias e >48 dias. Essas classes de intervalos são baseadas no intervalo entre cios normal de 21 dias com a variação de 18 24 dias.

\section{RESULTADOS}

Durante os 24 anos em que os dados foram coletados, ocorreram 1129 partos de 350 vacas. Do total, noventa por cento das vacas incluídas no estudo tiveram entre 2 e 5 partos e apenas $10 \%$ delas de 6 a 8 parições, com 40,6\% das vacas apresentando 2 partos e somente $2,9 \%$ atingindo oito partos.

A idade média ao primeiro parto ocorreu aos 37,1 meses (amplitude de 12,3 a 80,1 meses) e o primeiro cio aos 24,9 meses (amplitude de 13,8 a 70,6 meses), deduzindo-se que a concepção tenha ocorrido em torno dos 27,8 meses de idade (Tabela 1). A longevidade, considerando-se a idade ao úl-

Tabela 1 - Medidas centrais e de dispersão de características reprodutivas observadas num rebanho de 350 vacas de leite da raça Holandês, num período de 24 anos, pertencentes ao CPPSUL-EMBRAPA.

\begin{tabular}{lcrrrrr}
\hline \multicolumn{1}{c}{ Parâmetro } & $\mathrm{n}$ & Média & Mediana & Desvio Padrão & Máximo & Mínimo \\
& & & & & & \\
\hline & & & & & & \\
Idade ao primeiro cio (meses) & 266 & 24,9 & 24,7 & 5,2 & 70,6 & 13,8 \\
Idade ao primeiro parto (meses) & 348 & 37,1 & 35,0 & 8,7 & 80,1 & 12,3 \\
Intervalo parto primeiro cio (dias) & 783 & 97,0 & 81,0 & 67,5 & 568,0 & 11,0 \\
Intervalo entre cios (dias) & 1124 & 48,2 & 24,0 & 49,8 & 307,0 & 3,0 \\
Intervalo parto concepção (dias) & 785 & 150,7 & 114,0 & 106,2 & 757,0 & 11,0 \\
Intervalo entre partos (meses) & 772 & 14,6 & 13,2 & 3,8 & 34,7 & 9,8 \\
Número de crias & 1183 & 2,6 & 2,0 & 1,6 & 8,0 & 1,0 \\
& & & & & & \\
\hline
\end{tabular}

Ciência Rural, v. 31, n. 3, 2001. 
timo parto, foi 69,7 meses, em média, e não foi afetada pela ocorrência de doenças puerperais, nem pela ocorrência de mastite ( $\mathrm{P}>0,05)$. Em 48,1\% das gestações, foram verificados transtornos periparturientes, (aborto, natimorto, retenção de placenta, distocia e mastite) cujas freqüências estão representadas na tabela 2

O IPPC observado apresentou média de 97,0 dias, variando de 11 a 568 dias. Esse índice reprodutivo não foi afetado por nenhuma das alterações puerperais. Por outro lado, o IPC foi afetado pela ocorrência de distocia e natimortos. Os efeitos das alterações puerperais estão relacionados na tabela 3. A PL diária foi de $11,0 \mathrm{~kg}$ de leite, enquanto que a produção corrigida para 300 dias foi de $3306,9 \mathrm{~kg}$ (Tabela 4), sendo observada maior produção na oitava lactação, quando a produção média corrigida para 300 dias foi de 4212,0. A PL foi afetada significativamente por distocia $(\mathrm{P}<0,05)$. As vacas que apresentaram distocia produziram em média $2945,6 \mathrm{~kg}$ de leite e as que tiveram partos normais produziram $3326,1 \mathrm{~kg}$.

Foram também relacionadas as frequiências dos intervalos entre cios observados, representados na tabela 5. Esses cios foram estratificados em classes que permitiram inferir sobre problemas específicos tais como: liberação precoce de prostaglandina $\mathrm{F}_{2} \alpha$ (<17 dias); ciclos regulares (18 a 24); mortalidade embrionária (25 a 35 dias) e aborto (> 45 dias).

\section{DISCUSSÃO}

A idade à concepção das novilhas, em torno dos 27,8 meses, foi estimada tomando-se como

Tabela 2 - Transtornos periparturientes observados em 1181 gestações, observados em um rebanho leiteiro da raça Holandês, controlado durante 24 anos, pertencente a CPPSUL-EMBRAPA.

\begin{tabular}{lrc} 
Transtornos reprodutivos & $\mathrm{N}$ & Ocorrência por gestação \\
\hline & & \\
Aborto & 52 & 4,4 \\
Natimorto & 48 & 4,1 \\
Retenção de placenta & 132 & 11,2 \\
Mastite & 273 & 23,1 \\
Distocia & 63 & 5,3 \\
& & \\
Total & 568 & 48,1 \\
& &
\end{tabular}

base um período de gestação de 278 dias (McDONALD, 1989). A idade média ao primeiro parto dos animais relacionados neste trabalho ocorreu aos 37,1 meses, estando associada ao momento do primeiro serviço, realizado quando os animais alcançavam $340 \mathrm{~kg}$ de peso vivo. Essa idade pode ser considerada superior ao preconizado para o primeiro parto de vacas da raça Holandês, que, segundo FETROW et al. (1997), é de 22-25 meses. No entanto, há que se considerar que, no período de avaliação, os animais foram submetidos a diferentes sistemas de criação proporcionados pelos ensaios experimentais a que foram submetidos.

Dentre as afecções periparturientes, retenção de placenta e mastite foram as que ocorreram com maior frequiência. As demais anormalidades (aborto, natimortos e distocia) apresentaram freqüências inferiores a $10 \%$, o que estaria dentro de um limite aceitável (FETROW et al., 1997). Entretanto, a alta frequiência desses transtornos puerperais $(48,1 \%)$ indica falhas no manejo, o que dificulta a obtenção de melhores índices produtivos e reprodutivos. Os transtornos periparturientes ocorrem como um complexo e estão relacionados entre si, afetando outros parâmetros reprodutivos (CURTIS et al., 1985), sinalizando que medidas profiláticas reprodutivas e sanitárias para prevenir a ocorrência de um transtorno, podem diminuir o risco de incidência de outros relacionados direta ou indiretamente (STEVENSON \& CALL, 1988).

Tabela 4 - Produção de leite vitalícia $(\mathrm{kg})$ de 350 vacas leiteiras da raça Holandês do CPPSUL-EMBRAPA.

Produção de leite Média Desvio padrão Máximo Mínimo

\begin{tabular}{lrrrr}
\hline Corrigida 300 dias & 3306,9 & 964,3 & 7080 & 810 \\
Diária & 11,0 & 3,2 & 23,6 & 2,7 \\
& & & & \\
\hline
\end{tabular}


Tabela 5 - Freqüências dos intervalos entre cios observados em um rebanho leiteiro da raça Holandês, segundo a classificação de GAINES (1994).

Intervalos entre cios (dias)

Freqüência (\%)

$\begin{array}{cr}0-3 & 0,1 \\ 4-17 & 6,3 \\ 18-24 & 44,6 \\ 25-35 & 5,8 \\ 36-48 & 16,3 \\ >48 & 27,0\end{array}$

O IEP, cuja média foi de 14,6 meses, demonstra que o manejo reprodutivo desse rebanho estava aquém do ideal (12 meses) considerado por FERREIRA (1991), porém essa meta não é alcançada, sendo considerada apenas como um parâmetro indicador dos procedimentos de manejo reprodutivo.

As vacas que abortaram apresentaram um IEP 165,3 dias (5,5 meses) mais longo do que as que pariram normalmente. Esse valor foi tão alto, porque a uma gestação interrompida se sucede um puerpério anormal. Como a todo aborto segue-se uma inflamação endometrial, geralmente associada à retenção de placenta, pode haver um atraso na concepção seguinte (STEVENSON \& CALL, 1988). Neste trabalho, verificou-se esse efeito prejudicial do aborto sobre o IEP, porém, quando os casos registrados de retenção de placenta foram analisados isoladamente de aborto, não tiveram efeito sobre o IEP. Isso pode ter ocorrido porque o registro provavelmente atevese apenas ao processo patológico mais evidente, o aborto, ficando a retenção de placenta omitida, já que, segundo ROBERTS (1971), raramente a retenção de placenta ocorre de maneira isolada.

O acréscimo de 165,37 dias no IEP, em vacas que abortaram, significa a perda de uma produção de $1.189,1 \mathrm{~kg}$ de leite, que deixariam de ser acrescidos na receita da propriedade.

A ausência de efeitos da ocorrência de natimortos, mastite e distocia sobre o IEP difere de trabalhos que indicam um drástico efeito desses fatores sobre índices reprodutivos (ROBERTS, 1986; YOUNGQUIST, 1997). A natureza e subjetividade dos diagnósticos registrados nas fichas disponíveis podem ter contribuído para essa diferença.

O IPPC observado apresentou média de 97,0 dias. O valor mínimo de 11 dias ao primeiro cio observado pode ser considerado como falha no registro ou na observação, apesar de que intervalos menores (8 dias) têm sido registrados na bibliografia (SCHNEIDER et al. 1981). Esses dois problemas podem estar acontecendo de maneira simultânea e, por este trabalho se tratar de uma análise retrospectiva, não é possível se avaliar a veracidade dessa observação. $\mathrm{O}$ valor máximo observado não é comum (568 dias) e pode ser atribuído a falhas de observação ou prolongamento da estação reprodutiva. SCHNEIDER et al. (1981) documentaram 127 dias como máximo, o que fica ainda muito superior ao ideal recomendado por FETROW et al. (1997).

A variável IPPC foi menos sensível que o IPC, no que se refere aos efeitos das anormalidades puerperais. Isso, provavelmente, ocorre porque, mesmo com a ocorrência de problemas reprodutivos, a vaca pode apresentar o primeiro cio pós-parto sem que ocorra a concepção. Desordens como distocia e natimortos afetariam então o IPC, mesmo sem afetar o aparecimento do primeiro cio pós-parto. Por outro lado, o IPC foi afetado pela incidência de distocia e natimortos.

O IPC apresentou média de 150,7 dias, o que, segundo ESSLEMONT (1993) representa um problema grave. Esse mesmo autor considera ideal um IPC situado entre 75 e 85 dias, para que o IEP alcançado seja de 12 meses. Esse intervalo foi afetado significativamente por distocia $(\mathrm{P}<0,05)$, sendo de 186,0 dias para as que apresentaram distocia e 148,3 dias para as que tiveram parto normal. Cabe destacar que o manejo nutricional era o mesmo entre grupos. Os mecanismos envolvidos no retardo da concepção seguindo essas condições são desconhecidos, mas poderiam incluir o atraso da involução uterina (MORROW et al., 1969) e os efeitos do estresse sobre o eixo hipotálamo-hipófise, suprimindo a secreção de gonadotrofinas (LI \& WAGNER, 1983). Podem ser incluídas também como causas do atraso na concepção, inflamações genitais inespecíficas pós-puerperais, falta de controle da qualidade e aplicação do sêmen e ausência de registros ginecológicos periódicos. Vacas que apresentaram distocia tiveram um IPC 37,7 dias superior às que apresentaram partos normais. THOMPSON et al. (1983) mostraram que vacas com distocia tiveram intervalos parto primeiro serviço e parto concepção mais longos com maior número de serviços por concepção.

O IPC foi afetado significativamente $(\mathrm{P}<0,05)$ pela ocorrência de natimortos, com os casos positivos apresentando este intervalo 52,1 dias mais longo do que aqueles negativos. STEVENSON \& CALL (1988) não encontraram evidências de relação direta entre natimortos e performance reprodutiva. Entretanto, segundo esses mesmos autores, vacas com natimortos correm risco de apresentarem outras doenças, como prolapso de útero, retenção de placenta e, conseqüientemente, metrite, afetando assim indiretamente a eficiência reprodutiva. 
Os intervalos entre cios, observados neste trabalho (Tabela 5), indicam que em 16,4\% das ocasiões houve falha na detecção de cio. Possíveis alterações inflamatórias determinantes de liberação precoce de $\mathrm{PGF}_{2} \alpha$ ou falha na detecção de cio ocorreram em 6,3\% dos casos (intervalos entre 4-17 dias). Ciclos estrais regulares (18-24 dias) foram verificados em $44,6 \%$, salientando a importância da detecção de cio. Intervalos entre 25 e 35 dias sugerem mortalidade embrionária e foram verificados na freqüência de 5,8\%. Intervalos entre cios superiores a 48 dias (indicativo de aborto) ocorreram em 27,0\% dos casos. Caso se considere essa freqüência de intervalos entre cios superiores a 48 dias $(27,0 \%)$ como abortos, os registros de 4,4\% (Tabela 2) ficaram subdimensionados e sugerem falhas nos registros ou dificuldade de se diagnosticar o aborto a campo.

Propriedades que tenham deficiências na detecção de cio devem melhorar seu manejo, fazendo observações duas vezes ao dia ou utilizando de rufiões marcadores, ou ainda, através de programas de sincronização. A falha na detecção de cio contribui para o aumento do IPC e, subseqüentemente, o IEP, que foram significativamente correlacionados $(\mathrm{r}=0,32-\mathrm{P}<0,001)$.

Em 53,4\% das vacas, foi observado apenas um cio antes da concepção, o que representa um valor adequado, porém houve ocorrência de até 12 cios antes da concepção, sugerindo falhas de manejo que podem contribuir para um maior IPC. Relacionando-se a alta incidência de vacas que apresentaram apenas um cio antes da concepção com o alto IPC (150,7 dias), que é considerado como um problema severo dentro da propriedade, sinaliza-se para a necessidade de se atentar para falhas na detecção de cios.

O IEP e o IPC foram significativamente aumentados em consequiência de transtornos puerperais, prejudicando a eficiência reprodutiva. Embora os índices produtivos e reprodutivos gerais do rebanho não estivessem muito diferentes dos observados para rebanhos nacionais, como um IEP de 18 meses (FERREIRA, 1991), o manejo preventivo e o tratamento adequado de problemas puerperais podem determinar incrementos expressivos nesses índices.

A PL foi afetada significativamente $(\mathrm{P}<0,05)$ por distocia. As vacas que apresentaram distocia produziram, em média, $380,5 \mathrm{~kg}$ de leite a menos do que as que tiveram partos normais. Esses resultados se assemelham aos de SCHNEIDER $\boldsymbol{e t}$ al. (1981) em que vacas sem distocia produziram mais leite do que as vacas com distocia.
Deve-se ressaltar que os dados estudados pertencem a uma unidade experimental, não significando que representem a realidade encontrada em propriedades rurais, mas podem servir como base para que mais pesquisas sejam realizadas nesta área na qual poucos índices produtivos e reprodutivos são conhecidos.

\section{CONCLUSÕES}

Os índices IEP e IPC do rebanho são afetados por transtornos reprodutivos que ocorreram durante o puerpério, prejudicando a eficiência reprodutiva do rebanho. O monitoramento do IPC constitui uma importante ferramenta para o incremento da eficiência reprodutiva por estar intimamente associado ao IEP. A PL, por sua vez, foi afetada pela ocorrência de distocia, sugerindo o fator estressante que representa esse problema, refletindo-se na função lactogênica. A falha na detecção de cios reflete as falhas de manejo e registro de dados, comprometendo a eficiência reprodutiva do rebanho, prejudicando todos os índices produtivos.

\section{REFERÊNCIAS BIBLIOGRÁFICAS}

CURTIS, C.R., ERB, H.N., SNIFFEN, C.J. et al. Path analysis of dry period nutrition, postpartum metabolic and reproductive disorders, and mastitis in Holstein cows. J Dairy Sci, v.68, p.2347-2360, 1985

ESSLEMONT, R.J. Relationship between herd calving to conception interval and culling rate for failure to conceive. Vet Record v.133, p.163-164, 1993.

FERREIRA, A.M. Manejo reprodutivo e eficiência da atividade leiteira. Juiz de Fora, MG : EMPBRAPA, 1991. p.15. (Documento EMBRAPA, n.46).

FETROW, J., STEWART, S., EICKER, S. Reproductive health programs for dairy herds: Analysis of records for assessment of reproductive performance. In: YOUNGQUIST, R.S. Current therapy in large animal theriogenology. Philadelphia : Saunders, 1997. p.441-451.

GAINES, J.D. Proceedings for annual meeting. Kansas City : Society for Theriogenology, 1994. Analysis of reproductive efficiency of dairy herds: p.86-107.

LI, P.S., WAGNER W.C. Effects of hyperadrenal states on luteinizing hormone in cattle. Biol Reprod, v.29, p.11-16, 1983.

McDONALD, L.E. Pregnancy and parturition. In: McDONALD, L.E., PINEDA, M.H. Veterinary endocrinology and reproduction. Philadelphia. Lea \& Febiger, 1989. p.503-525.

MORROW, D.A., ROBERTS, S.J., McENTEE, K. A review of post partum ovarian activity and involution of the uterus and cervix in cattle. Cornell Vet, v.59, p.134, 1969. 
ROBERTS, S.J. Veterinary obstetrics and genital diseases, Michigan : Ann Arbor Edward Brothers, 1971. 776p.

ROBERTS, S.J. Veterinary obstetrics and genital diseases (Theriogenology). Michigan : Na Arbor Edwards Brothers, 1986. $981 \mathrm{p}$

SCHNEIDER, F., SHELFORD, R.G., PETERSON, R.G. $\boldsymbol{e}$ t al. Effects of early and late breeding of dairy cows on reproduction in current and subsequent lactation. J Dairy Sci, v.64, p.1996-2002, 1981
STEVENSON, J.S., CALL, E.P. Reproductive disorders in the periparturient dairy cow. J Dairy Sci, v.71, p.2572-2583, 1988

THOMPSON, J.R., POLLAK, E.J., PELISSIER, C.L. Interrelationships of parturition problems, production of subsequent lactation, reproduction and age at first calving. J Dairy Sci, v.66, p.1119-1127, 1983.

YOUNGQUIST, R.S. Current therapy in large animal theriogenology. Philadelphia : Saunders, 1997. 898p.

Ciência Rural, v. 31, n. 3, 2001. 